\title{
Treatment of severe depression - non-pharmacological aspects
}

\section{Richard Porter, Keith Linsley \& Nicol Ferrier}

Depression, in its severest forms, causes severe morbidity, has a high incidence of mortality and requires emergency treatment. In a previous article in this journal (Porter \& Ferrier, 1999), we discussed the emergency treatment of severe depression by pharmacological means and electroconvulsive therapy (ECT). Here, we will discuss the contribution of non-pharmacological aspects of management to fast, effective and safe treatment of severe depression. We will also complete our review of this subject by examining the efficacy of some emerging therapeutic interventions for severe depression.

Several problems make it difficult to determine the effectiveness of non-pharmacological interventions. The first is that studies that have examined effectiveness in 'severe' groups have used a variety of criteria or classifications to distinguish these patients from more mild cases. Groups that have been defined as severe include in-patients, those patients scoring above a set point on rating scales and patients meeting criteria for the endogenous or melancholic subtype of depression (Box 1). The second problem is that many of the possible interventions, such as admission to hospital or nursing observation, are difficult to study in a controlled way. The third is that some of the most important outcome measures, such as suicide, are rare. This makes it difficult to discriminate between treatments except in large studies.

\section{Treatment setting}

One of the first decisions to be made in management of a patient with severe depression is where they should be treated. In particular, it must be decided whether to admit patients to hospital. A large body of literature exists on alternatives to in-patient treatment in severe mental illness, but these studies do not establish whether relatively rare outcomes, such as suicide, increase if alternatives to hospitalisation are used and have generally not looked separately at outcomes in patients with affective disorder. Such studies have also found that certain patients still require admission. The factors that led to admission in the studies of Muijen et al (1992) and Dean \& Gadd (1990) are summarised in Box 2. This does not prove that admission is desirable in the presence of these factors, but indicates that even in the setting of a clinical trial, in which staff morale tends to be high and resources good, it is difficult to avoid admission. Therefore, it is probably useful to examine these factors when making the decision

Box 1 Research measures for severe depression

Three measures of severity have been used in research studies, based on:

- rating scales: usually $\leq 20$ on a 17 -item HRSD

- diagnostic criteria: melancholic (DSM-III, DSM-III-R, DSM-IV criteria) or endogenous (Research Diagnostic Criteria)

- in-patient status: a high percentage of patients with melancholia and suicidal ideation, and with social and family problems, are in-patients

Richard Porter is a senior lecturer in psychological medicine at the Christchurch School of Medicine, New Zealand. He has interests in affective disorder and is setting up a specialist affective disorders service in Christchurch. Keith Linsley is a consultant in general adult and community psychiatry at the County Hospital, Durham. He has a special interest in liaison psychiatry and suicide research. Nicol Ferrier is Head of Department at the Department of Psychiatry, University of Newcastle (Royal Victoria Infirmary, Queen Victoria Road, Newcastle upon Tyne, NE1 4LP). His research interests include the neurobiology and treatment of affective disorders, and he runs an academic and regional service for chronic affective disorders. 
Box 2 Factors that necessitated admission in clinical trials of out-patient alternatives

All patients

Severe psychosis

Violence

Delusions about the family

Pathological family dynamics

Assessment of the patient in police custody

Refusal to take prescribed drugs

Recurrent overdosing

Concurrent physical problems

Patients with depression

Psychosis

regarding admission. It is also interesting that they broadly correspond to factors that have emerged from studies of normal (i.e. non-trial) clinical practice. For instance, in a study of patients with depression in Denmark, in-patients had high rates of melancholia and suicidality but were not necessarily more severely depressed as measured by rating scales (Stage et al, 1998).

\section{The in-patient milieu}

While the aim of in-patient treatment may partly be to ensure safety while pharmacological treatment becomes effective, most services would aspire to the aims of practical and psychological help, education and rehabilition. Recent reports in the UK have in some cases been critical of in-patient services for failing to meet these aims. For instance, The Sainsbury Centre for Mental Health (1998) found that $40 \%$ of in-patients did not take part in any social or recreational activity during their stay in an acute psychiatric unit and that $30 \%$ did not take part in any therapeutic activity. Only 5\% received psychological therapies and nearly 50\% said they had not received enough information about their illness. There is evidence that educational packages are helpful in bipolar affective disorder (Perry et al, 1999) and improve compliance with medication, but no direct evidence of this in severe depression. There is little direct evidence regarding the therapeutic effects of simple supportive nursing or occupational therapy strategies in severe depression and these are areas in which further research would be helpful to allow us to plan services for this group.

Further factors that appear to be important in inpatient treatment are the connected issues of staff morale and staff attitudes to patients. It has been suggested that poor staff morale, or conflict among staff, can be a factor in hospital suicide. Morgan \& Priest (1984) found that staff had often been critical of patients who went on to commit suicide for being overdependent, provocative, unreasonable and pretending to have problems ("assuming disabilities"). This is further elaborated by Watts \& Morgan (1994), who coin the term 'malignant alienation' to describe the typical profile of relationships between patient and staff which may be a particular risk factor for future suicide. Such a situation can only be avoided by good staffing levels, high morale, good supervision and training and good teamwork (Morgan et al, 1998).

\section{Nursing observation}

Formalised nursing observation is usually concentrated on patients who are considered to be at high risk of suicide. As well as attempting to ensure safety of patients, formal observation may be a positive step, ensuring that suicidal patients and those with severe depression get one-to-one therapeutic involvement. It may, however, be experienced by patients as being a negative, passive or even punitive intrusion into their privacy and is sometimes delegated to less well-qualified staff who may be less capable of providing therapeutic input. A recent report (Department of Health, 1999) comments that:

"Observation is not simply a custodial activity. It is an opportunity for the nurse to interact in a therapeutic way with the patient on a one-to-one basis. Encouraging communication, listening, conveying to the patient that they are valued and cared for are important components of skilled nursing observations ... combined with a readiness to act."

The decision to institute observation is an important one and good communication is essential in making this decision. In a regional study of suicides, Blain \& Donaldson (1995) commented that, in some cases, concern regarding the possibility of suicide was reported in the notes but no action was taken. They suggest that enhancement of the level of observation in these cases may prevent suicides and that a lack of communication between medical and nursing staff may be a factor in some suicides. The National Confidential Inquiry (see Appleby, 2000) into homicides and suicides involving patients under psychiatric care found that suicide under intermediate levels of observation (e.g. observation every 10-15 minutes) is relatively common and suggest a review of such observation protocols. 


\section{Mental Health Act}

Few data are available regarding the impact of the use of the Mental Health Act, and randomised controlled trials are clearly impracticable. The possible negative effect of compulsory admission must be borne in mind. Harris \& Barraclough (1997) looked at the suicide risk in patients subject to involuntary admission and found a standardised mortality ratio (SMR) of 3852 (95\% CI 3328-4436), which compares to an SMR of 582 for all in-patients. This probably reflects the greater morbidity and disturbance in the former group, but nevertheless reminds us that use of the MHA certainly does not guarantee the safety of the patient. There is no good evidence that applies specifically to patients with affective disorder.

\section{Psychological therapies}

In addition to supportive psychotherapies undertaken by psychiatrists and other members of the multi-disciplinary team, specific manualised therapies are becoming more widely available and have been systematically evaluated. The important questions that need to be answered about such therapies are:

(a) whether in severe depression it is appropriate to use psychotherapies alone, without adjunctive pharmacotherapy;

(b) whether addition of specific psychotherapies to pharmacological management is routinely advantageous or useful only in specific situations.

The 'answers' are summarised in Box 3. However, an important issue to remember is that studies addressing this issue, particularly in in-patient treatment, nearly always compare the manualised therapy with some form of clinical management. In a well-run in-patient unit or community mental health team, clinical management may include a variety of supportive therapies conducted by welltrained, motivated staff. The lack of a demonstrable additional effect for a specific psychotherapy does not therefore prove the lack of a need for psychological issues to be addressed.

The best-researched therapies are cognitivebehavioural therapy (CBT) and interpersonal therapy (IPT). While both CBT and IPT have usually been found to be as effective as pharmacotherapy in out-patient depression, studies examining response in severe depression have yielded conflicting results. Four large studies have compared CBT with standard antidepressant medication in out-patients with severe depression and a meta-analysis of these has been conducted (DeRubeis et al, 1999). Severe depression was defined as a score of $>19$ on the 17item Hamilton Rating Scale for depression (HRSD; Hamilton, 1960) or score of $>29$ on the Beck Depression Inventory (Beck et al, 1961). CBT was used twice a week for the first 4-8 weeks. No statistically significant difference between the treatments, both of which were associated with improvement, was shown. Elkin et al (1989) also found IPT and pharmacotherapy to be equally effective in out-patients with severe depression. The evidence therefore suggests that, in severe depression, there is no significant difference in outcome between CBT or IPT and pharmacotherapy, and there was no consistent evidence of a difference in speed of onset of recovery.

In a meta-analysis of original data (595 outpatients with major depressive disorder treated in six similar studies), Thase et al (1997) examined the question of whether IPT or CBT alone are as effective as combination therapy. Treatment groups were CBT or IPT alone (psychotherapy alone; $n=243$ ) or IPT plus pharmacotherapy (combined therapy; $n=352$ ). While combined therapy was not significantly more effective than psychotherapy alone in milder depressions, a significant advantage was found for combined therapy in more severe and recurrent depressions. Therefore, although IPT or CBT alone appear as effective as pharmacotherapy alone in outpatients with severe depression, neither is as effective as a combination of the two.

In in-patient depression, it has been suggested that CBT can be easily adapted with an increased frequency of sessions to attempt to reduce hopelessness as quickly as possible, tailoring the programme

Box 3 Psychological therapies in emergency treatment

In severe depression (as measured by rating scales), melancholic depression and inpatient depression, psychotherapy alone cannot be recommended

Cognitive-behavioural or interpersonal therapy are useful adjuncts to pharmacological treatments in these cases, but it is not clear which patients should receive them

In every case, it is recommended that supportive and problem-solving psychotherapy, education, support for the family and opportunites to discuss social difficulties be available 
to varied levels of functioning and including significant others as collaborators (Stuart et al, 1997). Small, uncontrolled studies suggest that CBT alone can be effective in in-patients with depression (for a review, see Stuart et al, 1997), but to our knowledge, no studies compare in-patient CBT with standard pharmacotherapy. Therefore, at present, CBT alone for in-patients cannot be recommended. Neither is there good evidence regarding the relative efficacy of IPT alone in in-patients.

Evidence regarding the effectiveness of CBT and IPT in endogenous or melancholic depression is also conflicting. The large National Institute of Mental Health multi-centre trial (Elkin et al, 1989) studied 239 patients randomised to IPT, CBT, imipramine plus clinical management or placebo plus clinical management. Endogenous depression was present in $38 \%$ and was not a predictive factor in response to CBT, but did predict a better response to IPT. However, certain biological features (which are prevalent in the melancholic subtype of depression) may predict a relatively poor response to CBT or IPT. Notably, abnormal sleep profiles predict a poor response to both and hypercortisolaemia appears to predict a poor response to CBT (Thase \& Friedman, 1999).

Resources may limit the availability of specific therapies such as CBT and IPT. It is therefore important to examine whether adding these therapies to standard pharmacotherapy is effective and which patients might be targeted. Miller et al (1989) studied 45 in-patients with depression assigned either to standard treatment (hospitalmilieu therapy, pharmacotherapy or clinical management sessions) or to one of two brief psychotherapies (cognitive therapy or social skills training). All treatments began in the hospital and continued on an out-patient basis for 4 months after discharge. Significantly higher proportions of the patients who received additional psychotherapy of either kind had responded by the end of the formal treatment period and did not relapse for the remainder of the 1-year follow-up period. The advantages of cognitive therapy were only apparent in a 'high cognitive dysfunction' (high level of dysfunctional attitudes) group (Miller et al, 1990). In contrast, the NIMH study showed that more severe dysfunctional attitudes and beliefs predicted a poorer response to $\mathrm{CBT}$, while severe interpersonal difficulties predicted a poorer response to IPT (Sotsky et al, 1991).

There has been little research specifically into family interventions in severe depression. It is our practice to involve the family, especially when managing the transition from hospital to home. We also believe that where possible the family should be involved in education about the illness and its treatment.

\section{Suicide}

Inskip et al (1998) conclude that about $6 \%$ of patients with primary affective disorder eventually kill themselves. Suicide therefore constitutes an important and tragic outcome in severe depression. Nonpharmacological strategies for suicide prevention are summarised in Box 4.

Studies of all in-patient suicides have repeatedly failed to identify a constellation of risk factors that reliably predict suicide, and prediction of suicide in patients with affective disorders has proved similarly difficult. Goldstein et al (1991) prospectively followed 1906 patients with affective disorders for up to 13 years. Forty-six suicides occurred. Using multiple logistical regression, a model was created that identified the risk factors of male gender, previous suicide attempts (increasing with more attempts), suicidal ideas on admission, less favourable outcome at discharge and unipolar depression with family history of mania. When this model was used with a threshold of a $50 \%$ likelihood of suicide, it failed to predict any of the suicides.

The situation is further complicated by a number of conflicting studies. Modestin \& Kopp (1988) specifically examined suicides in in-patients with depression by comparing 75 suicides of patients with depression with 50 non-suicide controls with depression. The variables that emerged as discriminating best between the groups were male gender,

Box 4 Non-pharmacological interventions in suicide prevention

Careful assessment to establish diagnosis and comorbid conditions - particularly consider unipolar $v$. bipolar and screen for comorbid anxiety disorder or alcohol misuse

Admission and observation of high-risk patients

Increased support, observation and monitoring during risk periods - at night, early morning, immediately after admission, early in recovery and around the time of discharge

Advice on sleep hygiene and provision of a quiet, restful environment at night

Anxiety management strategies, discussion and monitoring of possible activating effects and of emergent anxiety with some antidepressants

Assess hopelessness and consider specific psychotherapy to address this 
suicidal behaviour at index admission and during hospitalisation, number of previous psychiatric hospitalisations, broken homes and disruption of close interpersonal relationships in the previous year. Previous studies also suggested that greater severity of depression (Barraclough et al, 1974) or psychotic depression (Roose et al, 1983) predisposed to suicide. In contrast, Fawcett et al (1990) reported a prospective study of 954 patients with affective disorder, of whom 569 had unipolar depression. Thirty-two patients committed suicide within 10 years. Risk factors for suicide within 1 year were anxiety, panic, insomnia, anhedonia, loss of concentration and alcohol misuse, while risk factors for later suicide were severe hopelessness, suicidal ideation and history of previous suicide attempts. A follow-up study, by the same group, of charts of patients for the week before suicide found that $64 \%$ denied suicidal ideation in their last communication in the week before suicide and $87 \%$ were judged to have severe anxiety/agitation. It is possible that those patients who planned an imminent suicide were less likely to communicate this to clinicians because of the likelihood that their plans would be thwarted. However, this evidence should not be interpreted as suggesting that assessment of suicidal ideation is unnecessary or that expression of suicidal ideation should not be taken seriously. It may be the case that, in suicide, victims' clues are given to family or friends rather than clinicians (Fawcett et al, 1969). Interviews with significant others and discussion of any possible communications that they may have had regarding suicide are therefore an important part of assessment of any patient with severe depression.

Further implications of these findings are that anxiety or panic may be an important feature of severe depression that predisposes to suicide. This may be particularly so since antidepressants can worsen these symptoms, especially early in the course of treatment. We suggest that these symptoms should be monitored carefully and that patients be warned of the possibility of initial worsening of their anxiety. Anxiety management strategies may be helpful.

Given the emergence of insomnia as a factor in suicide in depression, this may be an important target for careful monitoring and therapeutic intervention, both pharmacological and nonpharmacological. It also seems reasonable to suggest that in-patients with depression should be protected from undue disturbance at night. It has been suggested that early morning may be a time of particular risk, especially for patients with early morning wakening and diurnal variation in mood, and we suggest that clinicians routinely ask about suicidal thoughts in the early morning period (which may not be present when patients are interviewed later in the day). However, the Confidential Inquiry (Appleby, 2000) found in-patient suicides to be more common in the evening and at night and suggest enhanced observations at these times.

Hopelessness is an important feature of the presentation of depression - and an important focus for in-patient psychotherapies such as cognitive therapy. However, hopelessness emerged as a factor in later suicide rather than suicide within 1 year in the Fawcett et al (1990) study. Interesting data from a large study of suicide suggests that inter-episode measures of hopelessness, rather than hopelessness while depressed, or increase in hopelessness from baseline to a depressed state, may be the most important predictor of suicidal behaviour (Young et al, 1996). The implication of this is that it may be important to address the issue of hopelessness between episodes of depression in order to reduce suicidal behaviour during episodes.

Comorbid alcohol misuse appears to be a powerful additional factor in predisposing towards suicide (Cornelius et al, 1995) and in complicating the treatment of depression. Comorbidity is often specifically excluded from treatment studies, making it difficult to manage in an evidence-based way. All patients with depression should be screened for alcohol and drug misuse and given appropriate physical treatments and the opportunity for counselling.

It has been suggested that bipolar II disorder (depression with a history of hypomania but no mania) results in a higher incidence of suicide than either unipolar or bipolar depression. This may be because of its misdiagnosis as personality disorder, high rate of comorbidity or misdiagnosis as unipolar depression and treatment with antidepressants, which may induce rapid cycling or mixed states (for a review, see Rihmer \& Pestality, 1999). There is evidence that there is a high incidence of suicide where Research Diagnostic Criteria for both depression and mania are met concurrently (dysphoric mania - Dilsaver et al, 1994). We have previously discussed the specific pharmacological management of this situation (Porter \& Ferrier, 1999; Porter et al, 1999). There is little research on nonpharmacological aspects of management of bipolar depression or mixed states.

Finally, it may be helpful to examine in detail when in the course of the illness patients may be most likely to kill themselves. Clinical experience suggests that early reduction in retardation, in severe depression, may give patients the motivation that allows them to act on suicidal impulses or plans. We have discussed this in previous correspondence in this journal (Porter, 1999) and conclude that, while there is still no clear evidence for this phenomenon, patients should be very closely monitored early in a 
course of ECT or pharmacological treatment. In terms of stage of illness, the National Confidential Inquiry (Appleby, 2000) found that 23\% of in-patient suicides occurred within the first week of admission and $40 \%$ during the period when discharge was being planned. Twenty-four per cent of all inquiry cases occurred within 3 months of discharge. This pattern of high-risk periods is confirmed by other studies (Fawcett et al, 1990).

\section{Physical complications, exercise and emerging therapeutic interventions}

The physical consequences of depression are important both in the acute episode and long term. Physical examination and investigations are important because undiagnosed physical illnesses are common in psychiatric patients, may be the cause of their psychiatric symptoms and may result from their depressive illness. The long-term effects of depressive illness may include both cardiovascular disease and osteoporosis (Dinan, 1999), while the acute effects of depressive stupour can include dehydration, infection, decubitus ulcer and deep vein thrombosis (DVT). The careful assessment and investigation of patients with severe depression is therefore important and management should place emphasis on adequate hydration, nutrition and mobilisation. Care should be taken not to rehydrate patients with hyponatraemia (not uncommon with selective serotonin reuptake inhibitors (SSRIs) or carbamazepine) too rapidly as this can cause central pontine myelinosis (Lishman, 1998). Fluid balance charts should be instituted at an early stage and electrolytes checked regularly.

Exercise is important in avoiding acute complications such as DVT and, although we know of no evidence that it is effective in the treatment of severe depression, there are data suggesting a beneficial effect on mood and sleep. We suggest that an exercise programme be part of the activity schedule of inpatient units treating severe depression.

Another strategy that has seen a recent revival of interest is the use of sleep deprivation. This is of particular interest here because the effect occurs immediately (the day after sleep deprivation), is good in severe depression and better in endogenous than neurotic depression ( $70 \% v .48 \%$ response). A response has been seen even in patients with severe psychotic features (Wu \& Bunney, 1990). Unfortunately, there is a high relapse rate following sleep deprivation and this is higher if patients are not on medication ( $83 \%$ v. 59\%), making it important that it is only used as an adjunctive therapy. Some evidence suggests a role for a combination of serotonergic medication and repeated sleep deprivation. In patients with bipolar disorder experiencing an episode of depression, for instance, Benedetti et al (1999) showed a sustained response to 3 nights of sleep deprivation in patients on lithium.

Kripke (1998) identified six placebo-controlled studies of bright light therapy. In five of these, bright light was administered for 1 week, at the end of which there were significant improvements compared with placebo ranging from $12 \%$ to $35 \%$. Two of these studies used patients who were also on antidepressant medication and revealed an additive effect of administering both bright light and antidepressant medication. The one study lasting 4 weeks produced an improvement of $27 \%$ over placebo in patients already on antidepressant medication. The study with the greatest improvement was the combination of late-night sleep deprivation with bright light and medication, with the net benefit of bright light to placebo light of 35\% after 1 week (Neumeister et al, 1996). These approaches represent exciting developments that deserve further research, particularly given the fast speed of antidepressant effect reported. These treatments can, however, have adverse consequences - there have been reports of triggering mania and the energising of suicidal drive (Wu \& Bunney, 1990; Kripke, 1998). Careful supervision is advised if these therapies are considered.

\section{Conclusion}

Severe depression is a difficult condition to treat. Management requires several strategies and we have attempted to examine these in this and our previous article in this journal (Porter \& Ferrier, 1999). Nonpharmacological aspects of treatment that we consider to be particularly important are summarised in Box 5.

\section{References}

Appleby, L. (2000) Safer services: conclusions from the report of the National Confidential Inquiry. Advances in Psychiatric Treatment, 6, 5-15.

Barraclough, B., Bunch, J., Nelson, B., et al (1974) A hundred cases of suicide: clinical aspects. British Journal of Psychiatry, 125, 355-373.

Beck, A. T., Ward, C. H., Mendelson, M., et al (1961) An inventory for measuring depression. Archives of General Psychiatry, 4, 561-571.

Benedetti, F., Colombo, C., Barbini, B., et al (1999) Ongoing lithium treatment prevents relapse after total sleep deprivation. Journal of Clinical Psychopharmacology, 19, 240-245. 
Box 5 Important non-pharmacological aspects of treatment

Careful consideration of the setting in which treatment should take place and of the need for compulsory admission

Frequent assessment of suicide risk and physical state, good communication between staff and therapeutic observation by welltrained staff where necessary

A range of therapeutic skills available on inpatient wards, including practical, psychological and occupational support; staff providing this should be well trained and supervised in order to recognise and deal with negative countertransference

Consideration of specific psychological therapies such as IPT or CBT in selected cases

Further research into therapies that may accelerate response, such as bright light or sleep deprivation

Careful discharge planning with good communication between staff and with the family of patients

Blain, P. A. \& Donaldson, L. J. (1995) The reporting of inpatient suicides: identifying the problem. Public Health, 109, 293-301.

Cornelius, J. R., Salloum, I. M., Mezzich, J., et al (1995) Disproportionate suicidality in patients with comorbid major depression and alcoholism. American Journal of Psychiatry, 152, 358-364.

Dean, C. \& Gadd, E. M. (1990) Home treatment for acute psychiatric illness. British Medical Journal, 301, 1021-1023.

Department of Health (1999) Practice Guidance: Safe and Supportive Observation of Patients at Risk. Mental Health Nursing: 'Addressing Acute Concerns'. London: Standing Nursing and Midwifery Advisory Committee, Department of Health, England and Wales.

DeRubeis, R. J., Gelfand, L. A., Tang, T. Z., et al (1999) Medications versus cognitive behavior therapy for severely depressed outpatients: mega-analysis of four randomized comparisons. American Journal of Psychiatry, 156, 1007-1013.

Dilsaver, S. C., Chen, Y. W., Swann, A. C., et al (1994) Suicidality in patients with pure and depressive mania. American Journal of Psychiatry, 151, 1312-1315.

Dinan, T. G. (1999) The physical consequences of depressive illness (editorial). British Medical Journal, 318, 826.

Elkin, I., Shea, M. T., Watkins, J. T., et al (1989) National Institute of Mental Health Treatment of Depression Collaborative Research Program. General effectiveness of treatments. Archives of General Psychiatry, 46, 971-982; discussion, 983.

Fawcett, J., Leff, M. \& Bunney, W. E. Jr (1969) Suicide. Clues from interpersonal communication. Archives of General Psychiatry, 21, 129-137.

-, Scheftner, W. A., Fogg, L., et al (1990) Time-related predictors of suicide in major affective disorder. American Journal of Psychiatry, 147, 1189-1194.

Goldstein, R. B., Black, D. W., Nasrallah, A., et al (1991) The prediction of suicide. Sensitivity, specificity, and predictive value of a multivariate model applied to suicide among 1906 patients with affective disorders. Archives of General Psychiatry, 48, 418-422.
Hamilton, M. (1960) A rating scale for depression. Journal of Neurology, Neurosurgery and Psychiatry, 23, 56-62.

Harris, E. C. \& Barraclough, B. (1997) Suicide as an outcome for mental disorders. A meta-analysis. British Journal of Psychiatry, 170, 205-228.

Inskip, H. M., Harris, E. C. \& Barraclough, B.(1998) Lifetime risk of suicide for affective disorder, alcoholism and schizophrenia. British Journal of Psychiatry, 172, 35-37.

Kripke, D. F. (1998) Light treatment for nonseasonal depression: speed, efficacy, and combined treatment. Journal of Affective Disorders, 49, 109-117.

Lishman, W. A. (1998) Organic Psychiatry: The Psychological Consequences of Cerebral Disorder (3rd edn). Oxford: Blackwell Science.

Miller, I. W., Norman, W. H. \& Keitner, G. I. (1989) Cognitivebehavioral treatment of depressed inpatients: six- and twelve-month follow-up. American Journal of Psychiatry, 146, 1274-1279.

- — - \& - (1990) Treatment response of high cognitive dysfunction depressed inpatients. Comprehensive Psychiatry, 31, 62-71.

Modestin, J. \& Kopp, W. (1988) Study on suicide in depressed inpatients. Journal of Affective Disorders, 15, 157-162.

Morgan, H. G. \& Priest, P. (1984) Assessment of suicide risk in psychiatric in-patients. British Journal of Psychiatry, 145, 467-469.

—, Buckley, C. \& Nowers, M. (1998) Face to face with the suicidal. Advances in Psychiatric Treatment, 4, 188-196.

Muijen, M., Marks, I., Connolly, J., et al (1992) Home based care and standard hospital care for patients with severe mental illness: a randomised controlled trial. British Medical Journal, 304, 749-754.

Neumeister, A., Goessler R., Lucht, M., et al (1996) Bright light therapy stabilizes the antidepressant effect of partial sleep deprivation. Biological Psychiatry, 39, 16-21.

Perry, A., Tarrier, N., Morriss, R., et al (1999) Randomised controlled trial of efficacy of teaching patients with bipolar disorder to identify early symptoms of relapse and obtain treatment. British Medical Journal, 318, 149-153.

Porter, R. J. (1999) Changes in suicidal ideation and psychomotor retardation during electroconvulsive therapy (letter). Advances in Psychiatric Treatment, 5, 398.

— \& Ferrier, N. (1999) Emergency treatment of depression. Advances in Psychiatric Treatment, 5, 3-10.

-, Ferrier, N. \& Ashton, H. (1999) Anticonvulsants as mood stabilisers. Advances in Psychiatric Treatment, 5, 96-103.

Rihmer, Z. \& Pestality, P. (1999) Bipolar II disorder and suicidal behavior. Psychiatric Clinics of North America, 22, 667-673.

Roose, S. P., Glassman, A. H., Walsh, B. T., et al (1983) Depression, delusions, and suicide. American Journal of Psychiatry, 140, 1159-1162.

Sainsbury Centre for Mental Health (1998) Acute Problems: A Survey of the Quality of Care in Acute Psychiatric Wards. London: Sainsbury Centre for Mental Health.

Sotsky, S. M., Glass, D. R., Shea, M. T., et al (1991) Patient predictors of response to psychotherapy and pharmacotherapy: findings in the NIMH Treatment of Depression Collaborative Research Program. American Journal of Psychiatry, 148, 997-1008.

Stage, K. B., Bech, P., Gram, L. F., et al (1998) Are in-patient depressives more often of the melancholic subtype? Danish University Antidepressant Group. Acta Psychiatrica Scandinavica, 98, 432-436.

Stuart, S., Wright, J. H., Thase, M. E., et al (1997) Cognitive therapy with inpatients. General Hospital Psychiatry, 19, 42-50.

Thase, M. E. \& Friedman, E. S. (1999) Is psychotherapy an effective treatment for melancholia and other severe depressive states? Journal of Affective Disorders, 54, 1-19.

-, Greenhouse, J. B., Frank, E., et al (1997) Treatment of major depression with psychotherapy or psychotherapypharmacotherapy combinations. Archives of General Psychiatry, 54, 1009-1015.

Watts, D. \& Morgan, G. (1994) Malignant alienation. Dangers for patients who are hard to like. British Journal of Psychiatry, 164, 11-15. 
Wu, J. C. \& Bunney, W. E. (1990) The biological basis of an antidepressant response to sleep deprivation and relapse: review and hypothesis. American Journal of Psychiatry, 147, $14-21$.

Young, M. A., Fogg, L. F., Scheftner, W., et al (1996) Stable trait components of hopelessness: baseline and sensitivity to depression. Journal of Abnormal Psychology, 105, 155-165.

\section{Multiple choice questions}

1. Cognitive-behavioural therapy:

a can be effective in out-patient depression

b has a faster onset of action than pharmacotherapy in in-patient depression

$c$ is contra-indicated in in-patient depression

$\mathrm{d}$ is more effective than antidepressant pharmacotherapy in severe depression (score $>19$ on the 17-item HRSD).

2. Suicide:

a can be accurately predicted in in-patient depression b may be more likely when staff do not like the patient

c in patients with depression, is associated with sleep disturbance

$\mathrm{d}$ is common around the time of discharge.

3. The acute physical consequences of severe depression include:

a osteoporosis

b deep venous thrombosis

c dehydration

d dental sepsis.

\section{MCQ answers}


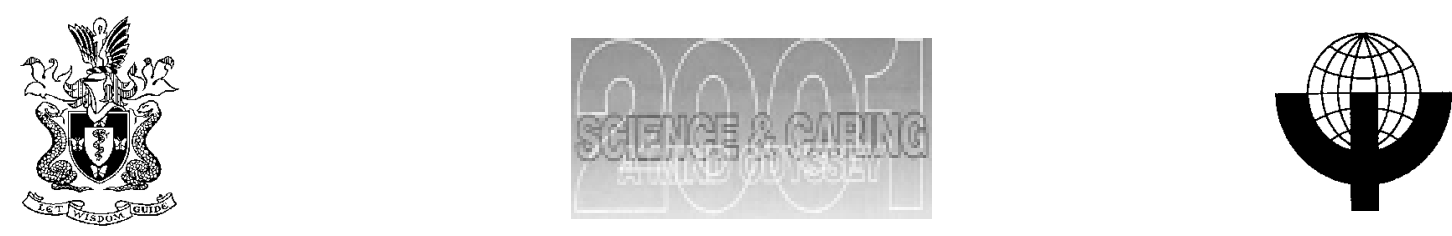

The Royal College of Psychiatrists Annual Meeting World Psychiatric Association European Regional Meeting

9-13 July 2001

Queen Elizabeth II Conference Centre, London

\section{Five reasons for embarking on 200 I A Mind Odyssey}

- Five days of enjoyable, effective and motivating presentations, tapping into an international skills base

- Self-select thematic pathways through each day's programme, connecting questions and answers across European boundaries

- Interactive sessions and workshops using different techniques to deliver top-quality learning experiences for today's needs

- A rewarding experience for trainees! The meeting has been specially planned to be of value to trainees; in addition they will benefit from a reduced delegate fee and, to mark its launch, some free places on a 'teaching the teachers' course, successful completion of which will count towards a postgraduate qualification

- The launch of '200I A Mind Odyssey', a year of media events raising awareness of artistic expression in mental health - its importance, its benefits and its power. This celebration of the arts and the mind will involve practitioners, users and carers, linking to national and international groups to effect a groundbreaking change in the image and perception of mental health

Conference Office, Royal College of Psychiatrists, I7 Belgrave Square, London SWIX 8PG Telephone +44 (0)20 7235 235I, ext. I42; fax +44 (0)20 72596507

The programme and registration form can be viewed at: www.rcpsych.ac.uk/200I/index.htm 